\title{
Supporting Information: Isothermal Chemical Denaturation: Data Analysis, Error Detection and Correction by PARAFAC2
}

Dillen Augustijna , Alina Kulakova ${ }^{b}$, Sujata Mahapatra ${ }^{b, c}$, Pernille Harris ${ }^{b}$, Åsmund Rinnan ${ }^{a}{ }^{*}$

a. Department of Food Science, Faculty of Life Sciences, University of Copenhagen, Rolighedsvej 30, DK-1958 Frederiksberg C, Denmark.

b. Department of Chemistry, Technical University of Denmark, Kemitorvet 207, 2800 Kgs. Lyngby, Denmark.

c. Novozymes A/S, Biologiens Vej 2, 2800 Kongens Lyngby, Denmark

*Corresponding author, aar@food.ku.dk

\section{Abstract}

The following document contains a selection of figures, illustrating the raw data input, scaled raw data for comparison of peaks shape and shift, and as validation of the models represented in the manuscript. In the manuscript, figures are used where outliers have been removed to improve clarity. For completeness, outliers are shown in all figures in the supplementary materials. Additionally, a GIF file is available separately, as part of the supplementary materials. The raw data is to be made publicly available in the future.

Index

1 Raw data \& effect of formulation on tryptophan peak shape

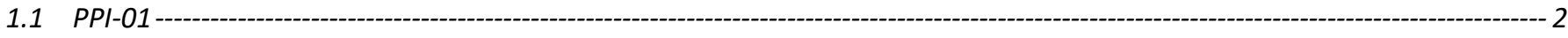

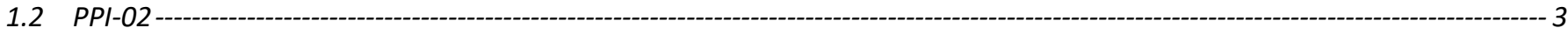

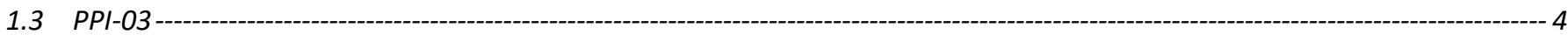

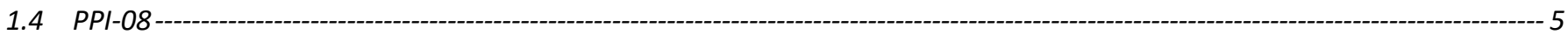

1.5 PPI-10-- 6

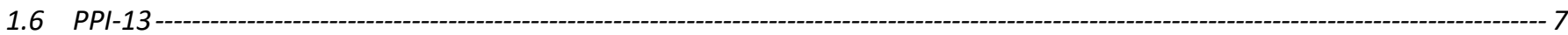

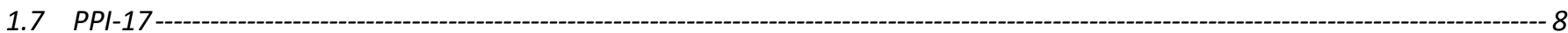

2 PARAFAC2 models \& core Consistency-- 9

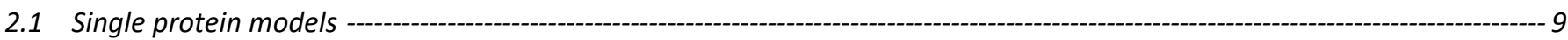

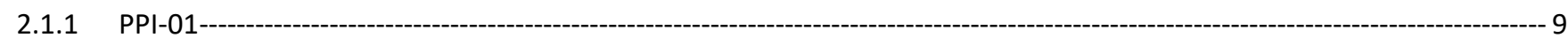

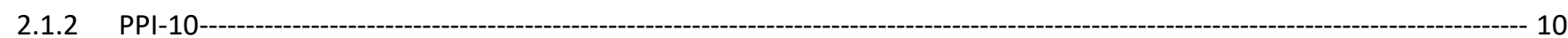

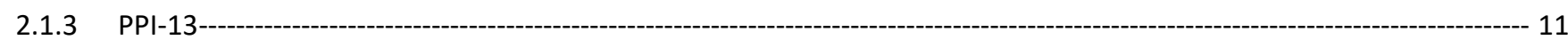

2.2 Combined protein models --

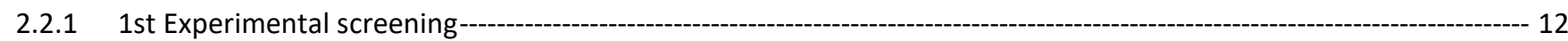

2.2.2 2nd Experimental screening---1--- 


\section{S1 Raw data \& effect of formulation on tryptophan peak shape}

\section{S1.1 PPI-01}

A

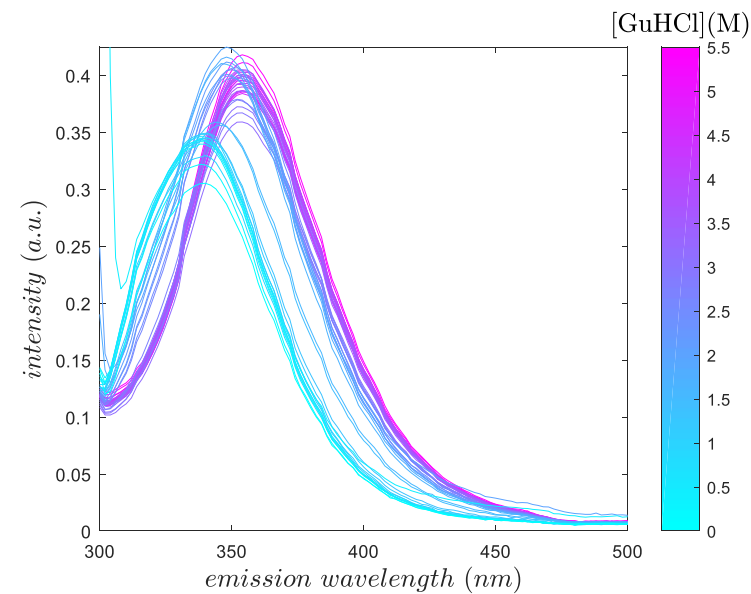

C

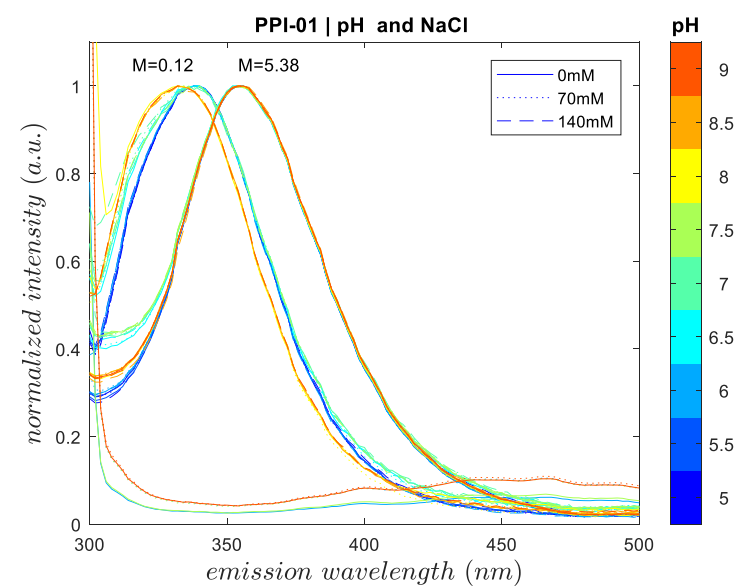

B

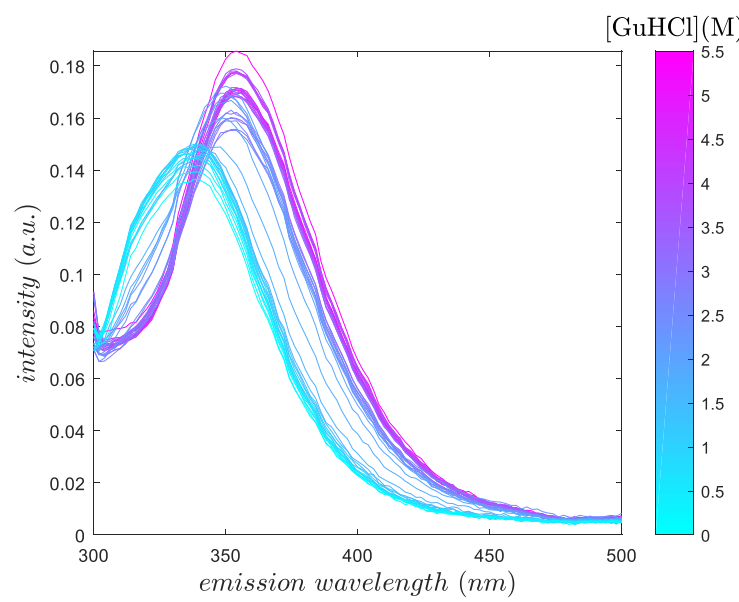

D

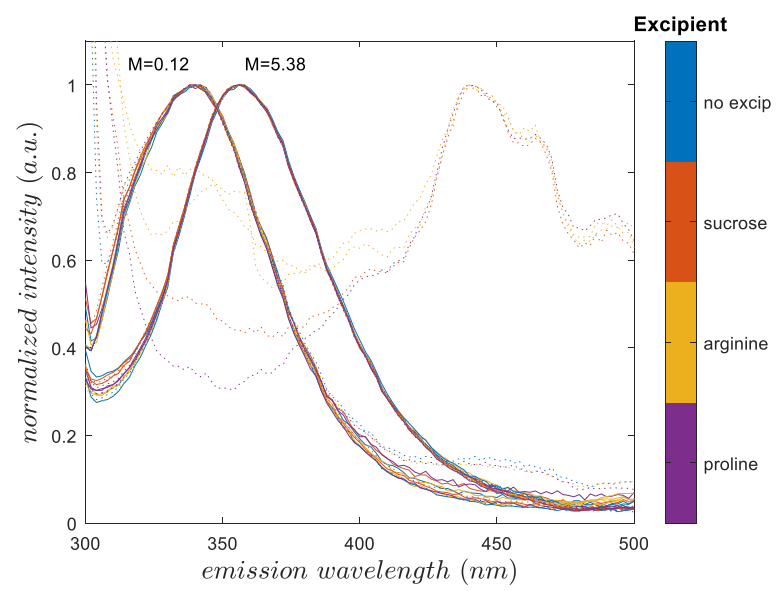

Figure S 1-1: Raw data \& effect of formulation on tryptophan peak shape for PPI-01. Two formulation unfolding patterns are shown from the first experimental series over $\mathrm{pH}$ and $\mathrm{NaCl}$ concentrations. The figure is analogous to Figure 2 of the manuscript, but outliers have not been removed. A) \& B) shows emission spectra measured in wells at different denaturant concentration, formulated at A) pH 5.0 with $0 \mathrm{mM} \mathrm{NaCl,}$ and B) $\mathrm{pH} 6.5$ with $140 \mathrm{nM} \mathrm{NaCl}$. C) \& D) shows emission spectra from two denaturation concentrations, $0.12 \mathrm{M}$ and $5.38 \mathrm{M} \mathrm{GuHCl}$. C) shows the emission spectra of the $1^{\text {st }}$ experimental series colored according to $\mathrm{pH}$, while $\mathrm{D}$ ) are emission spectra from the $2^{\text {nd }}$ experimental series, colored according to excipient. 
A

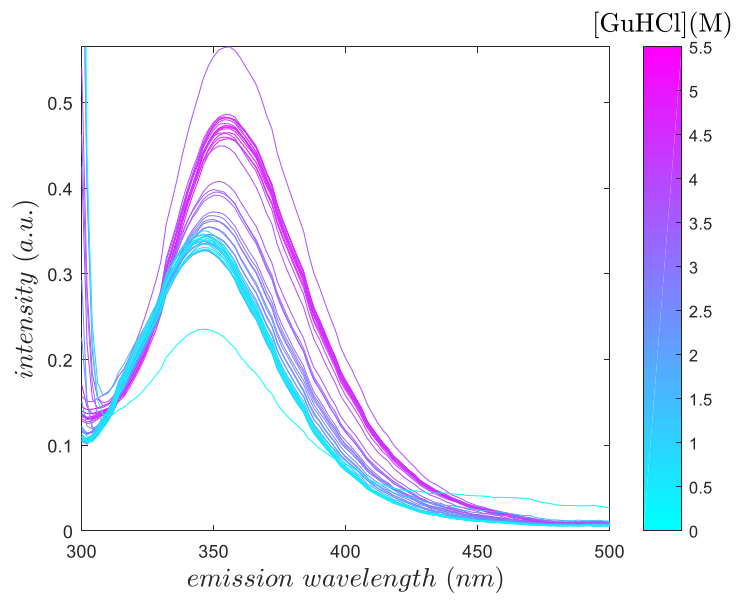

C

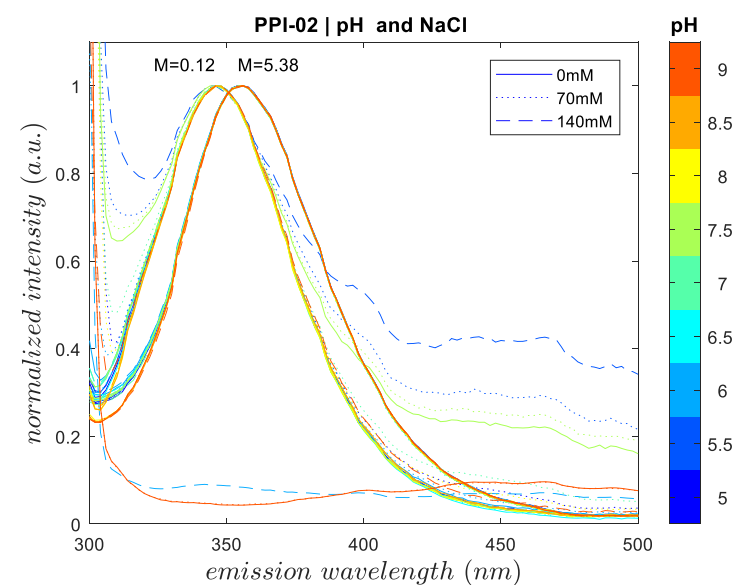

B

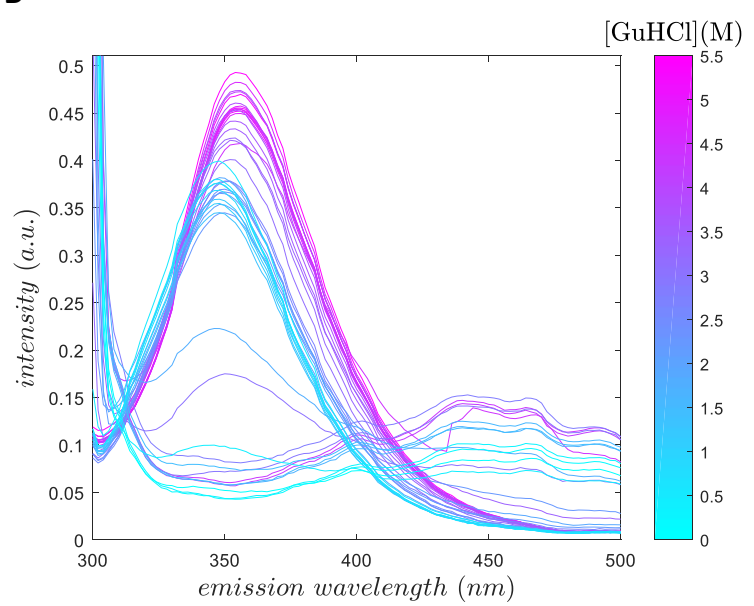

D

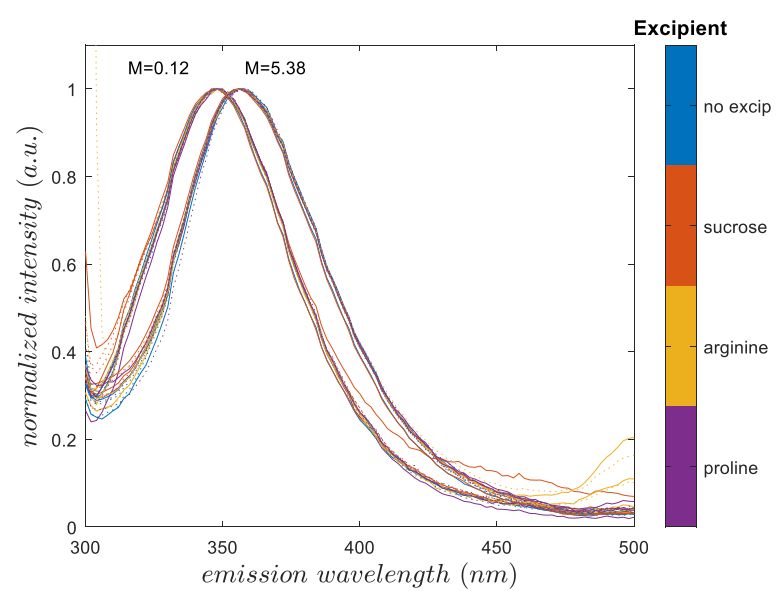

Figure S 1-2: Raw data \& effect of formulation on tryptophan peak shape for PPI-02. Two formulation unfolding patterns are shown from the first experimental series over $\mathrm{pH}$ and $\mathrm{NaCl}$ concentrations. The figure is analogous to Figure 2 of the manuscript, but outliers have not been removed. A) \& B) shows emission spectra measured in wells at different denaturant concentration, formulated at A) pH 5.0 with $0 \mathrm{mM} \mathrm{NaCl,}$ and B) $\mathrm{pH} 6.5$ with $140 \mathrm{nM} \mathrm{NaCl}$. C) \& D) shows emission spectra from two denaturation concentrations, $0.12 \mathrm{M}$ and $5.38 \mathrm{M} \mathrm{GuHCl}$. C) shows the emission spectra of the $1^{\text {st }}$ experimental series colored according to $\mathrm{pH}$, while $\mathrm{D}$ ) are emission spectra from the $2^{\text {nd }}$ experimental series, colored according to excipient. 


\section{S1.3 PPI-03}

A

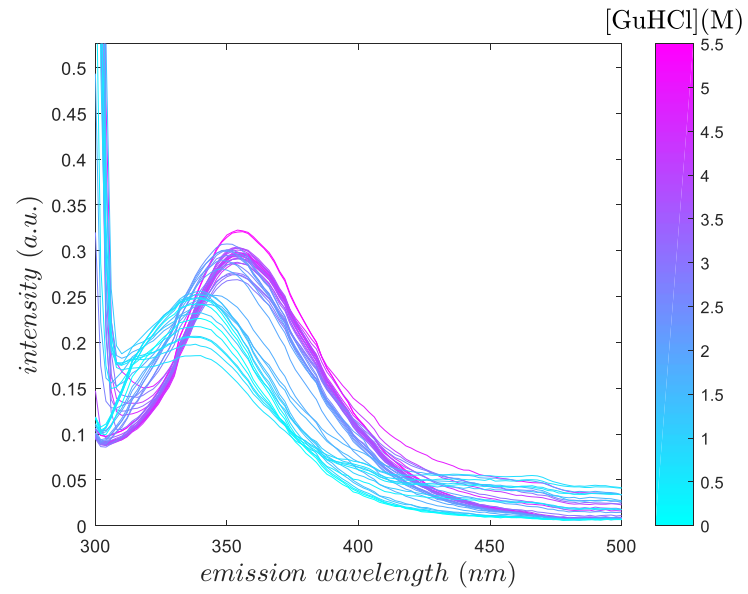

C

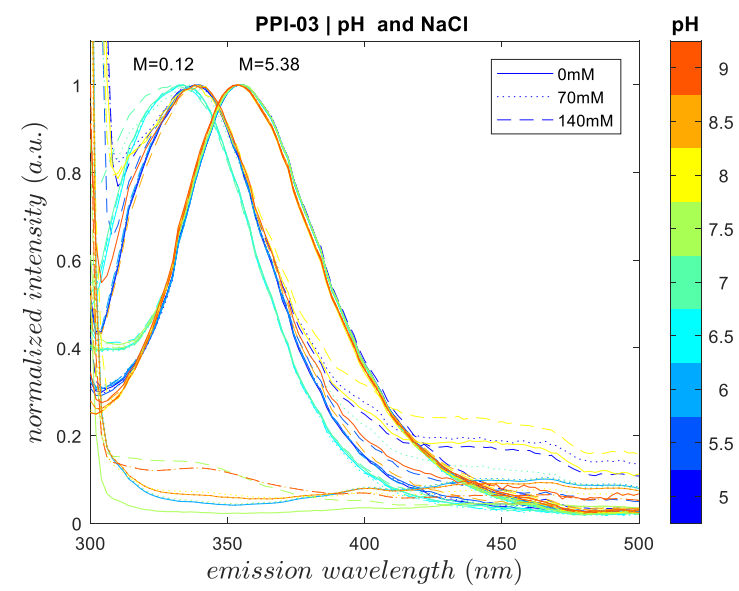

B

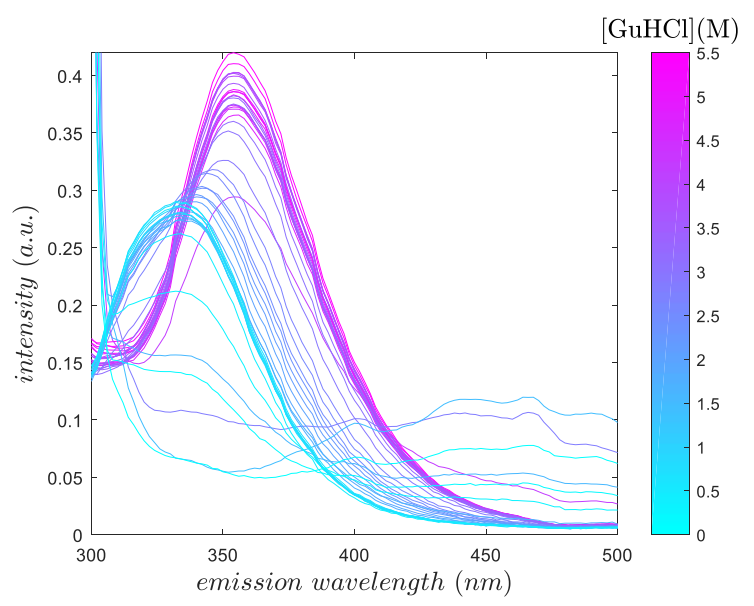

D

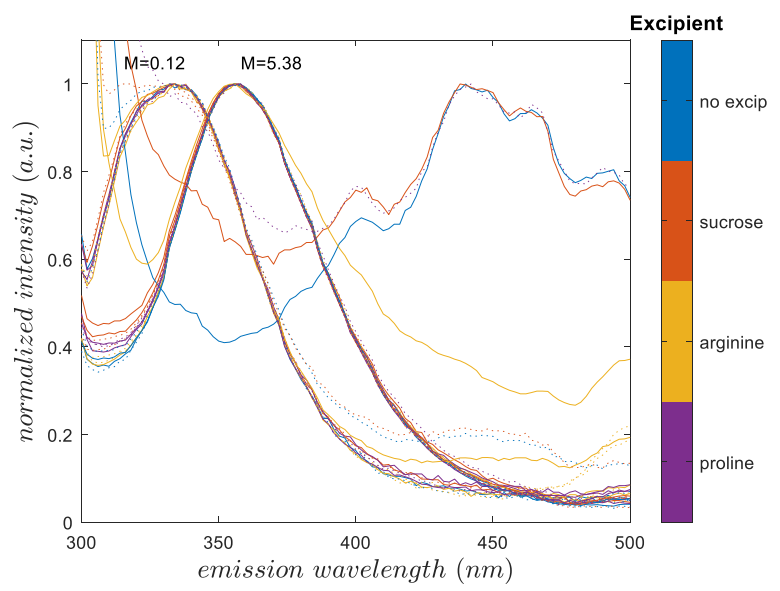

Figure S 1-3: Raw data \& effect of formulation on tryptophan peak shape for PPI-03. Two formulation unfolding patterns are shown from the first experimental series over $\mathrm{pH}$ and $\mathrm{NaCl}$ concentrations. The figure is analogous to Figure 2 of the manuscript, but outliers have not been removed. A) \& B) shows emission spectra measured in wells at different denaturant concentration, formulated at A) pH 5.0 with $0 \mathrm{mM} \mathrm{NaCl}$, and B) $\mathrm{pH} 6.5$ with $140 \mathrm{nM} \mathrm{NaCl}$. C) \& D) shows emission spectra from two denaturation concentrations, $0.12 \mathrm{M}$ and $5.38 \mathrm{M} \mathrm{GuHCl}$. C) shows the emission spectra of the $1^{\text {st }}$ experimental series colored according to $\mathrm{pH}$, while $\mathrm{D}$ ) are emission spectra from the $2^{\text {nd }}$ experimental series, colored according to excipient. 
A

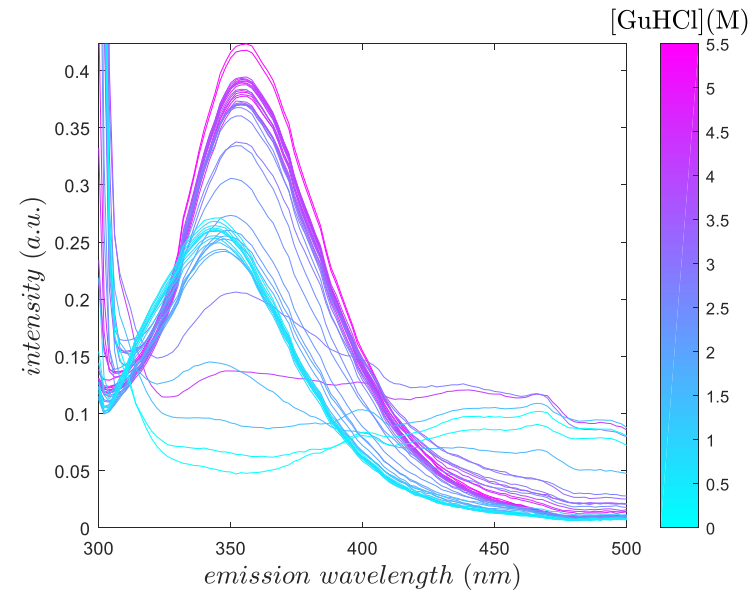

C

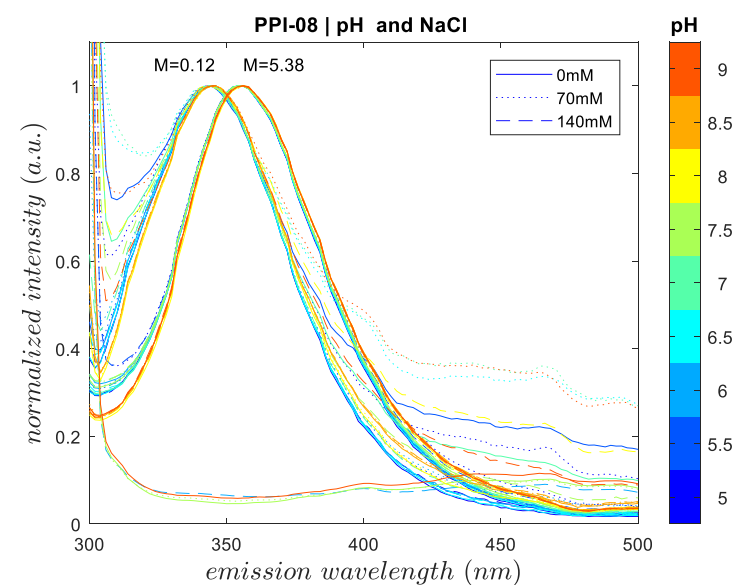

B

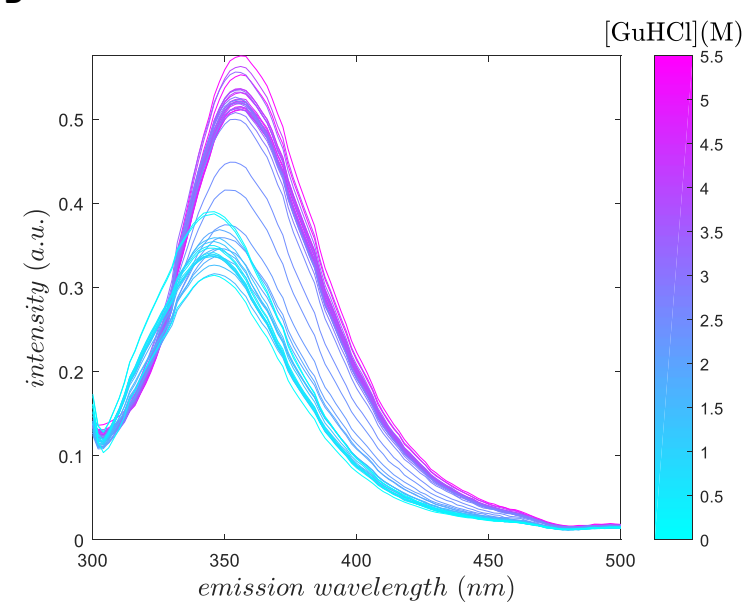

D

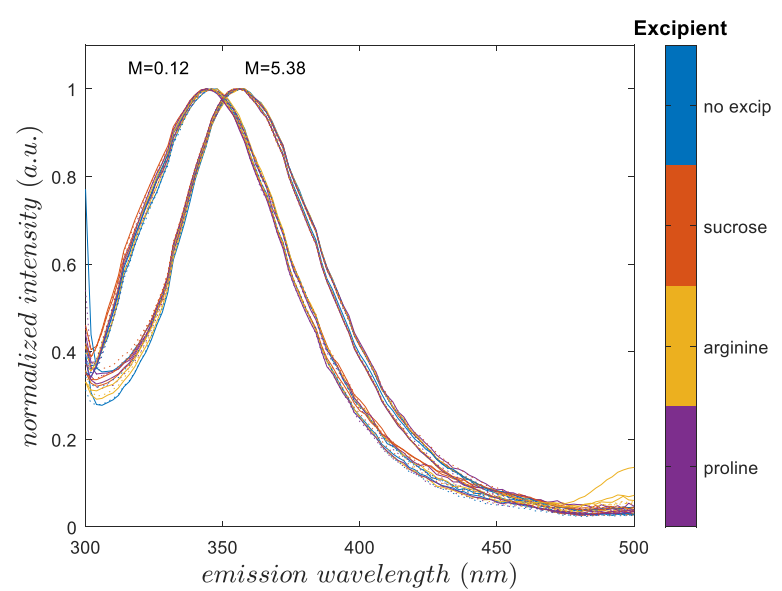

Figure S 1-4: Raw data \& effect of formulation on tryptophan peak shape for PPI-08. Two formulation unfolding patterns are shown from the first experimental series over $\mathrm{pH}$ and $\mathrm{NaCl}$ concentrations. The figure is analogous to Figure 2 of the manuscript, but outliers have not been removed. A) \& B) shows emission spectra measured in wells at different denaturant concentration, formulated at A) pH 5.0 with $0 \mathrm{mM} \mathrm{NaCl,}$ and B) $\mathrm{pH} 6.5$ with $140 \mathrm{nM} \mathrm{NaCl}$. C) \& D) shows emission spectra from two denaturation concentrations, $0.12 \mathrm{M}$ and $5.38 \mathrm{M} \mathrm{GuHCl}$. C) shows the emission spectra of the $1^{\text {st }}$ experimental series colored according to $\mathrm{pH}$, while $\mathrm{D}$ ) are emission spectra from the $2^{\text {nd }}$ experimental series, colored according to excipient. 
A

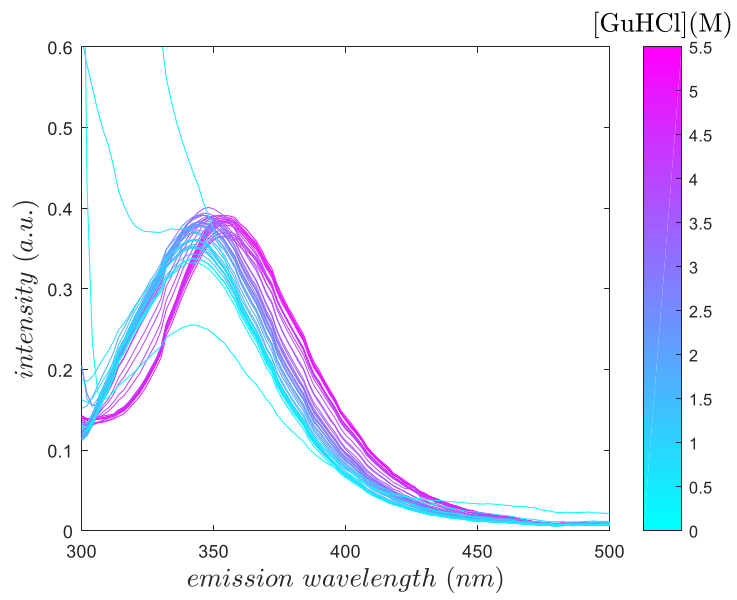

C

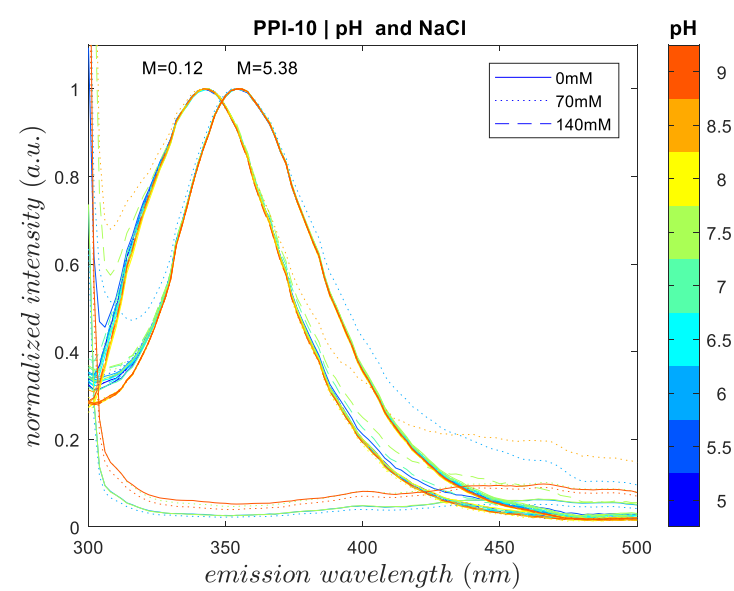

B

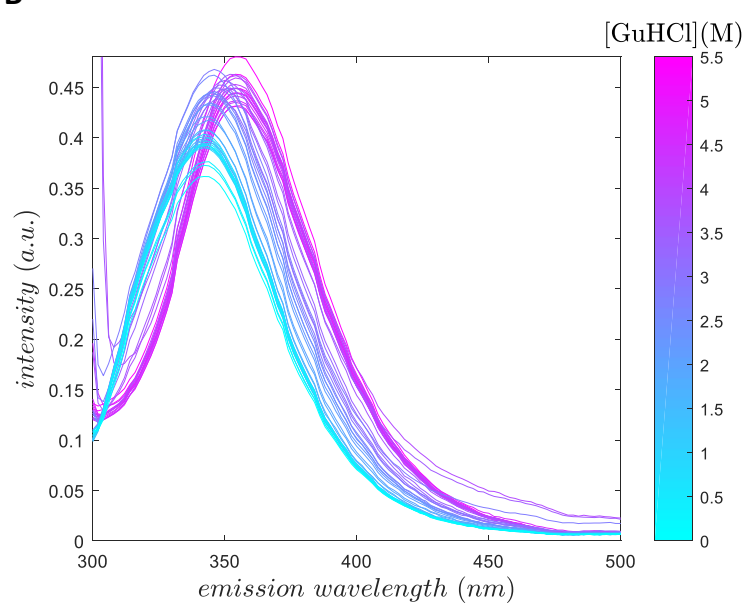

D

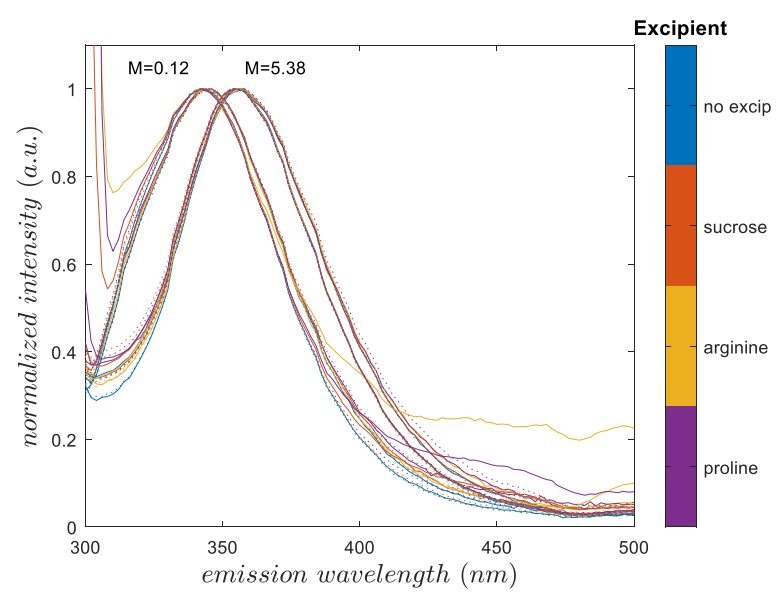

E

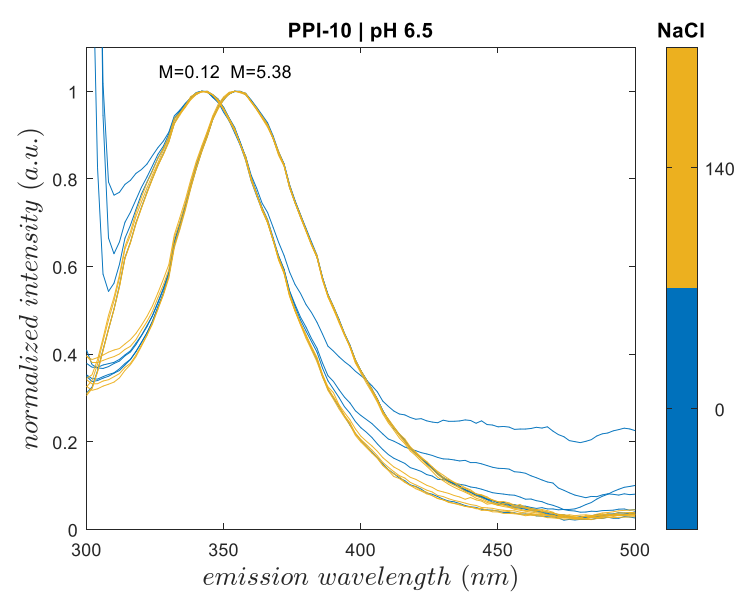

Figure S 1-5: Raw data \& effect of formulation on tryptophan peak shape for PPI-10. Two formulation unfolding patterns are shown from the first experimental series over $\mathrm{pH}$ and $\mathrm{NaCl}$ concentrations. The figure is analogous to Figure 2 of the manuscript, but outliers have not been removed. A) \& B) shows emission spectra measured in wells at different denaturant concentration, formulated at A) pH 5.0 with $0 \mathrm{mM} \mathrm{NaCl,}$ and B) $\mathrm{pH} 6.5$ with $140 \mathrm{nM} \mathrm{NaCl}$. C), D) \& E) shows emission spectra from two denaturation concentrations, $0.12 \mathrm{M}$ and $5.38 \mathrm{M} \mathrm{GuHCl}$. C) shows the emission spectra of the $1^{\text {st }}$ experimental series colored according to $\mathrm{pH}, \mathrm{D}$ ) are emission spectra from the $2^{\text {nd }}$ experimental series, colored according to excipient while $E$ ) is a copy of the emission spectra from D), but colored according to $\mathrm{NaCl}$ concentration. 
A

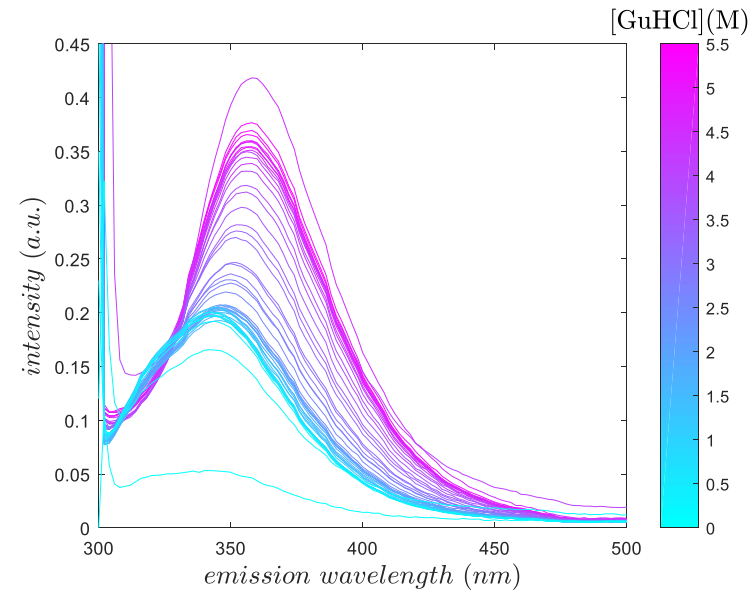

C

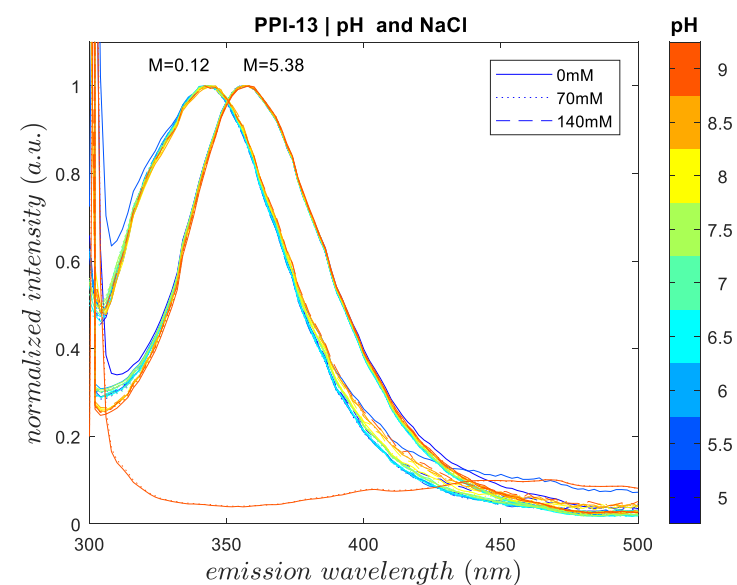

A

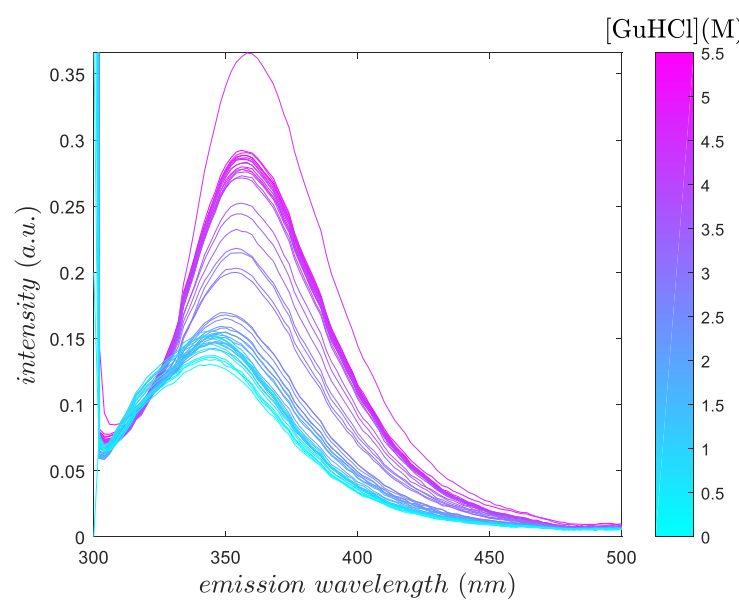

D

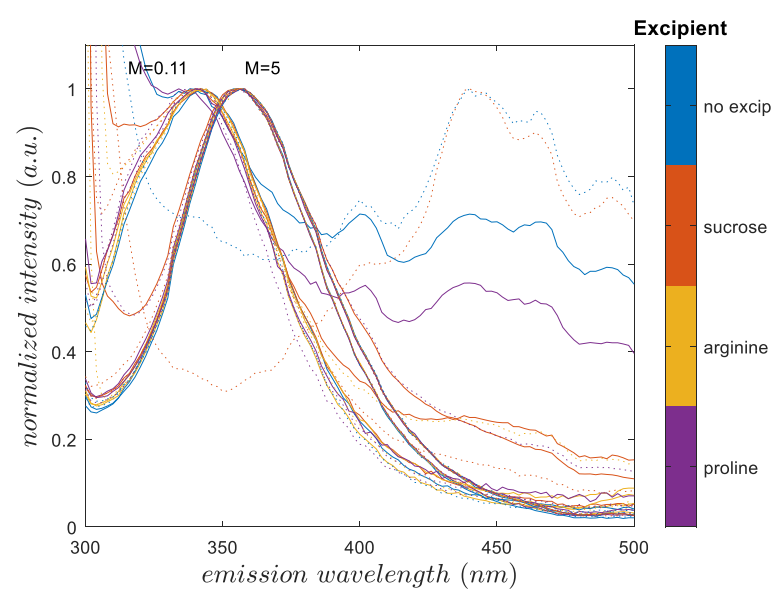

Figure S 1-6: Raw data \& effect of formulation on tryptophan peak shape for PPI-13. Two formulation unfolding patterns are shown from the first experimental series over $\mathrm{pH}$ and $\mathrm{NaCl}$ concentrations. The figure is analogous to Figure 2 of the manuscript, but outliers have not been removed. A) \& B) shows emission spectra measured in wells at different denaturant concentration, formulated at A) pH 5.0 with $0 \mathrm{mM} \mathrm{NaCl,}$ and B) $\mathrm{pH} 6.5$ with $140 \mathrm{nM} \mathrm{NaCl}$. C) \& D) shows emission spectra from two denaturation concentrations, $0.12 \mathrm{M}$ and $5.38 \mathrm{M} \mathrm{GuHCl}$. C) shows the emission spectra of the $1^{\text {st }}$ experimental series colored according to $\mathrm{pH}$, while $\mathrm{D}$ ) are emission spectra from the $2^{\text {nd }}$ experimental series, colored according to excipient. 
A

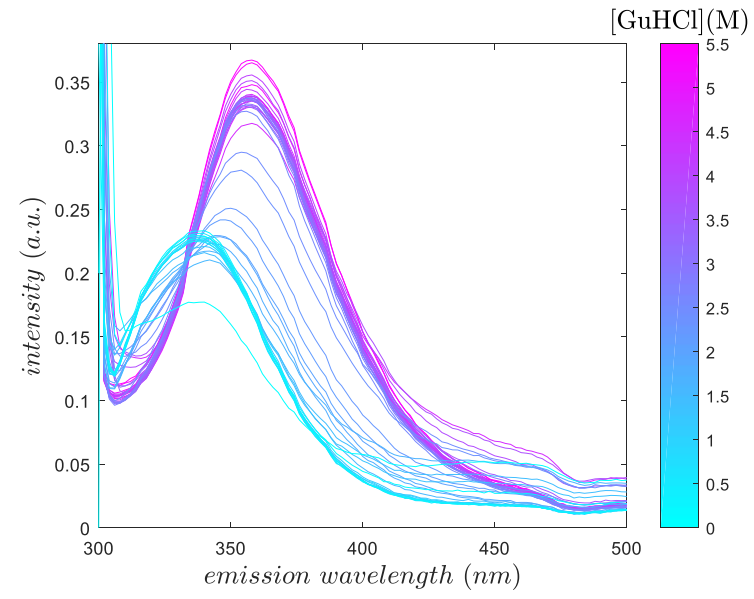

C

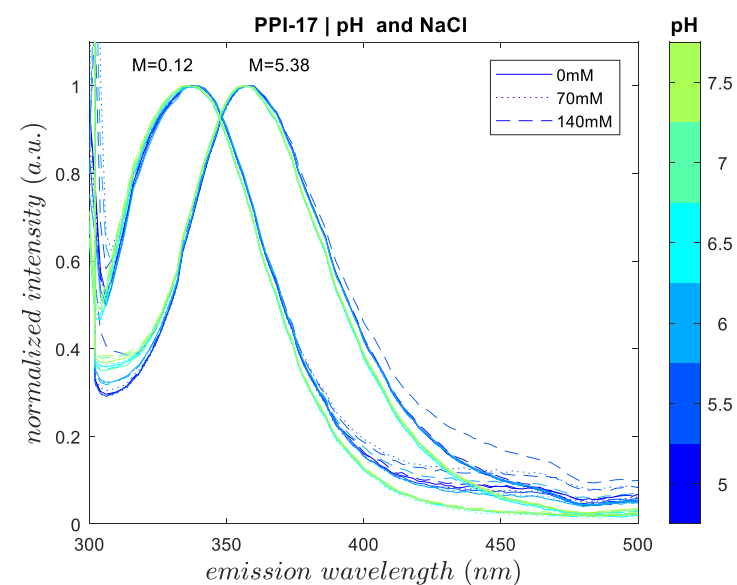

A

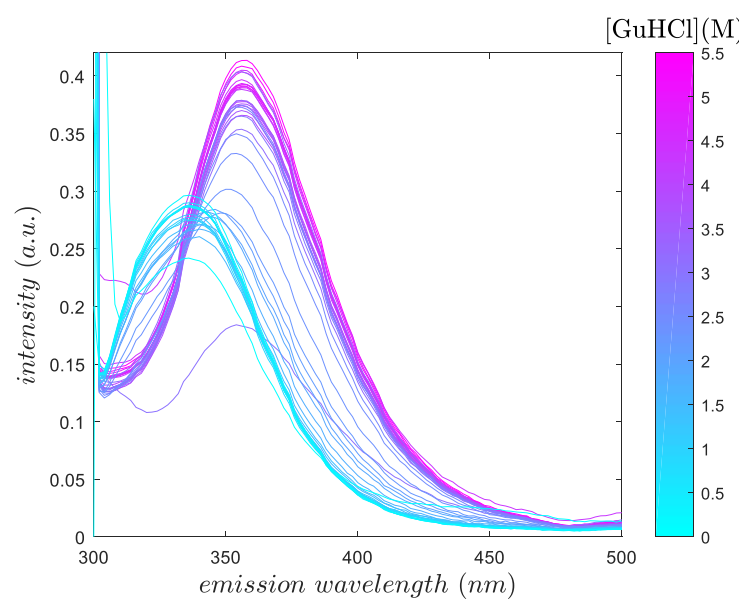

D

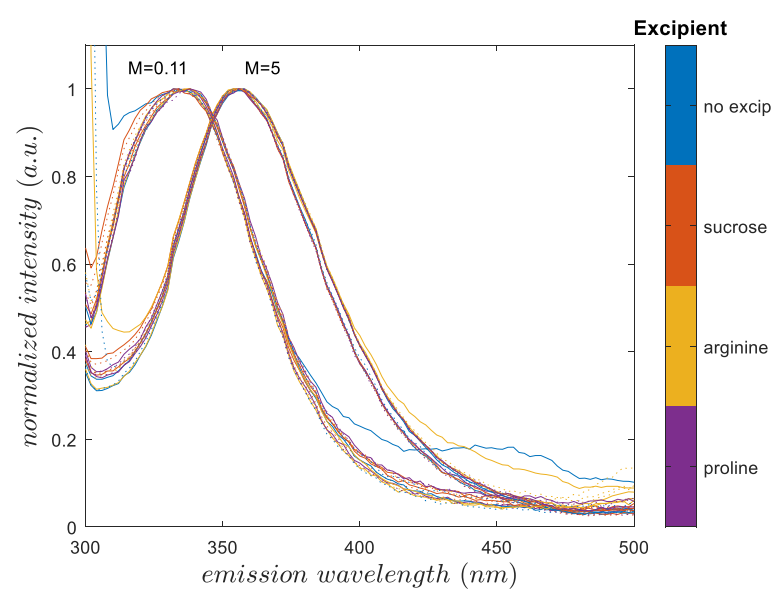

Figure S 1-7: Raw data \& effect of formulation on tryptophan peak shape for PPI-17. Two formulation unfolding patterns are shown from the first experimental series over $\mathrm{pH}$ and $\mathrm{NaCl}$ concentrations. The figure is analogous to Figure 2 of the manuscript, but outliers have not been removed. A) \& B) shows emission spectra measured in wells at different denaturant concentration, formulated at A) pH 5.0 with $0 \mathrm{mM} \mathrm{NaCl,}$ and B) $\mathrm{pH} 6.5$ with $140 \mathrm{nM} \mathrm{NaCl}$. C) \& D) shows emission spectra from two denaturation concentrations, $0.12 \mathrm{M}$ and $5.38 \mathrm{M} \mathrm{GuHCl}$. C) shows the emission spectra of the $1^{\text {st }}$ experimental series colored according to $\mathrm{pH}$, while $\mathrm{D}$ ) are emission spectra from the $2^{\text {nd }}$ experimental series, colored according to excipient. 


\section{S2 PARAFAC2 models \& core Consistency}

\section{S2.1 Single protein models}

\section{S2.1.1 PPI-01}

A

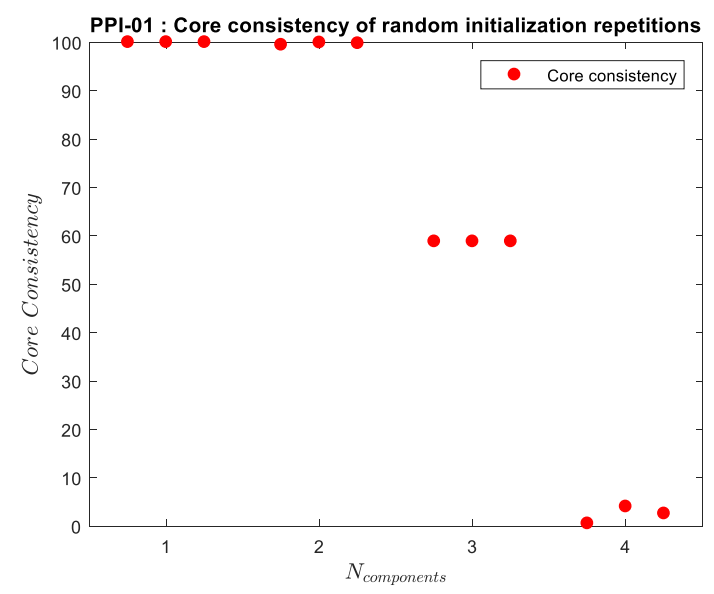

C

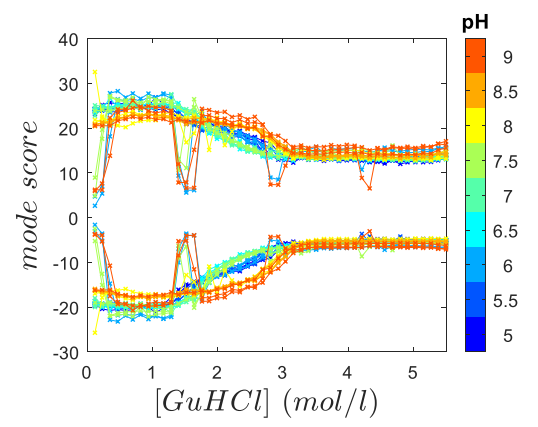

B
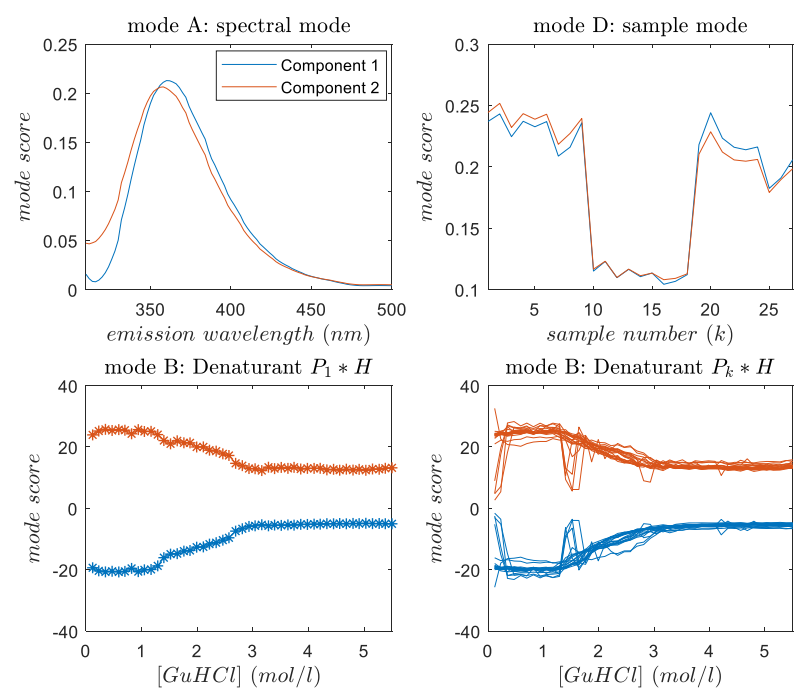

D

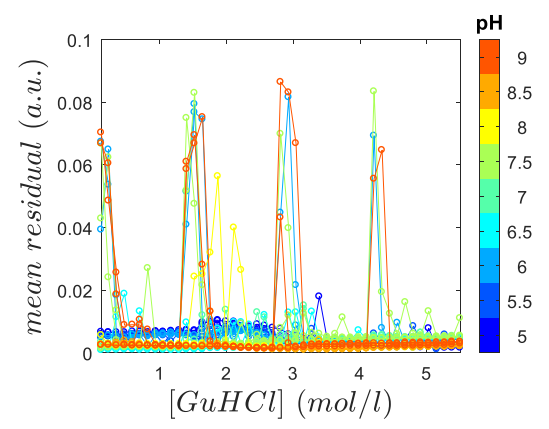

Figure S 2-1: Shows the results of PARAFAC2 modelling for PPI-01 from the $1^{\text {st }}$ experimental series. A) the core consistency as a function of number of components, B) all modes of the PARAFAC2 model, $C$ ) mode $D$ scores colored according to $p H$ and $D$ ) the residual at each denaturation concentration, colored according to $\mathrm{pH}$. D) indicates $6.9 \%$ outliers. 
S2.1.2 PPI-10

A

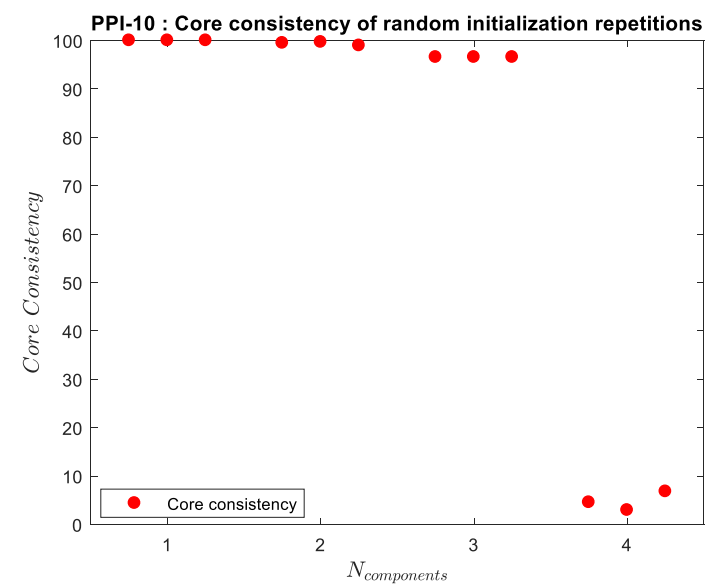

C

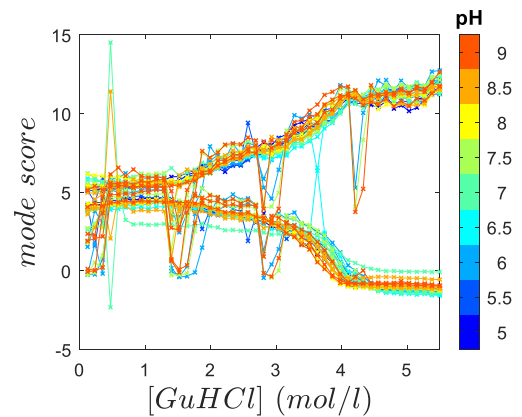

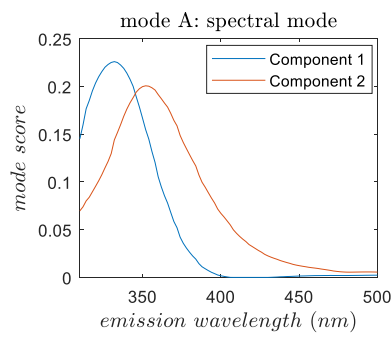
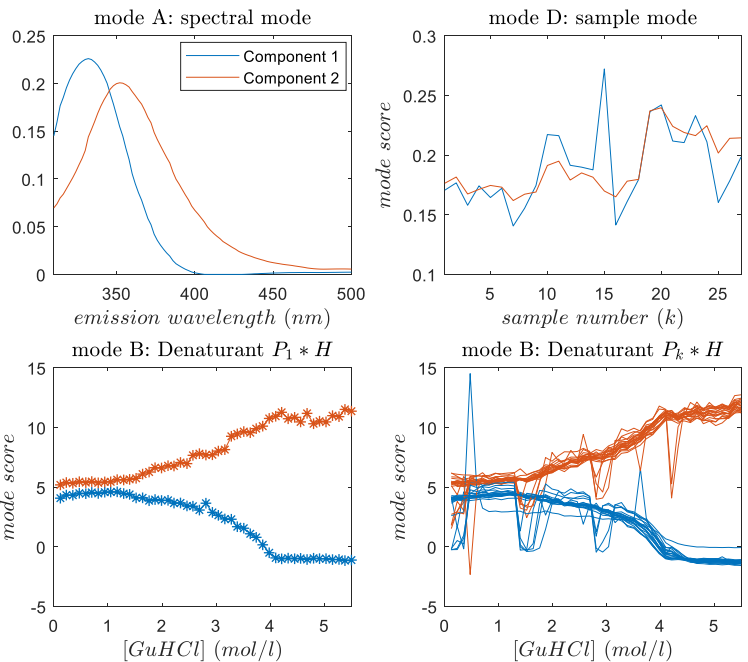

D

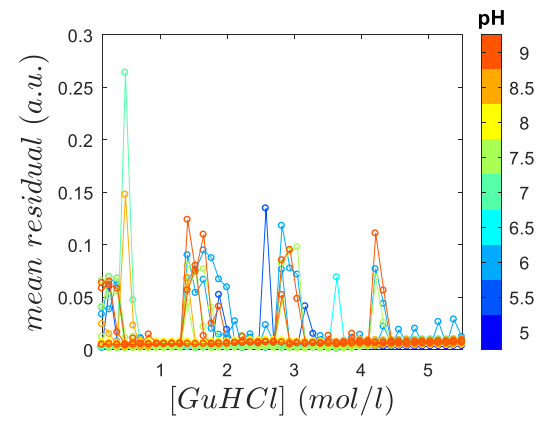

Figure S 2-2: Shows the results of PARAFAC2 modelling for PPI-10 from the $1^{\text {st }}$ experimental series. A) the core consistency as a function of number of components, $B$ ) all modes of the PARAFAC2 model, C) mode $D$ scores colored according to $p H$ and $D$ ) the residual at each denaturation concentration, colored according to $\mathrm{pH}$. $\mathrm{D}$ ) indicates $8.4 \%$ outliers. 
A

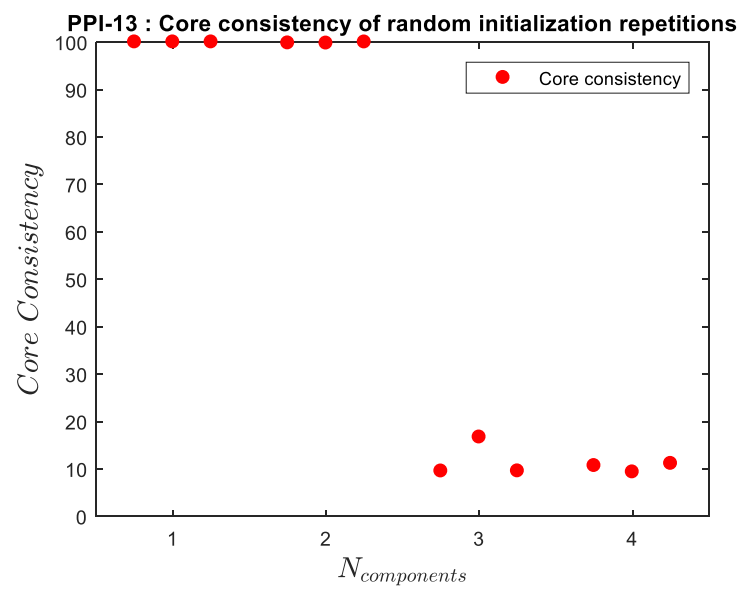

C

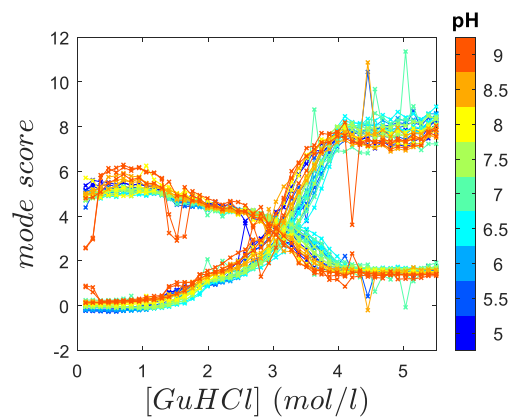

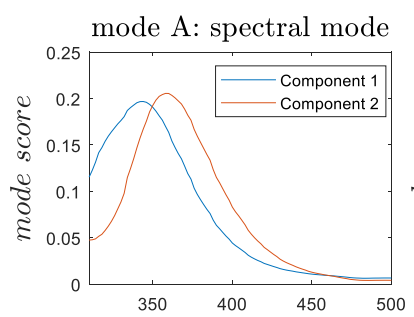

emission wavelength $(\mathrm{nm})$ mode B: Denaturant $P_{1} * H$

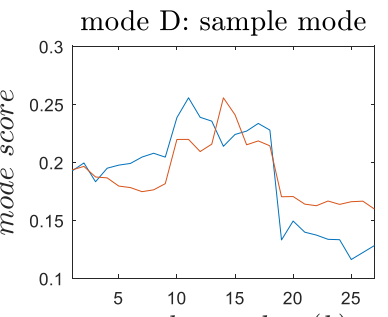

sample number $(k)$
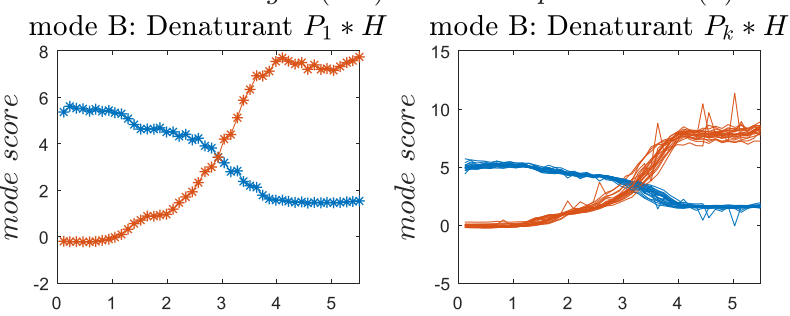

$[\mathrm{GuHCl}](\mathrm{mol} / \mathrm{l})$

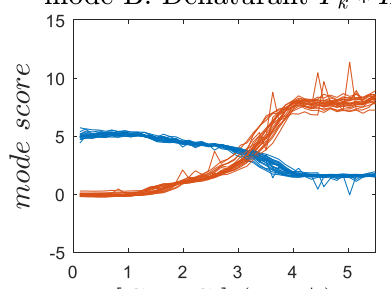

$[\mathrm{GuHCl}](\mathrm{mol} / \mathrm{l})$

D

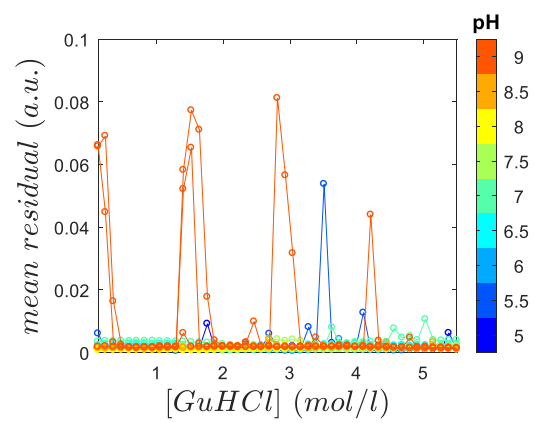

Figure S 2-3: Shows the results of PARAFAC2 modelling for PPI-13 from the $1^{\text {st }}$ experimental series. A) the core consistency as a function of number of components, B) all modes of the PARAFAC2 model, C) mode D scores colored according to $\mathrm{pH}$ and D) the residual at each denaturation concentration, colored according to $\mathrm{pH}$. D) indicates $6.1 \%$ outliers. 


\section{S2.2 Combined protein models}

\section{S2.2.1 1st Experimental screening}

A

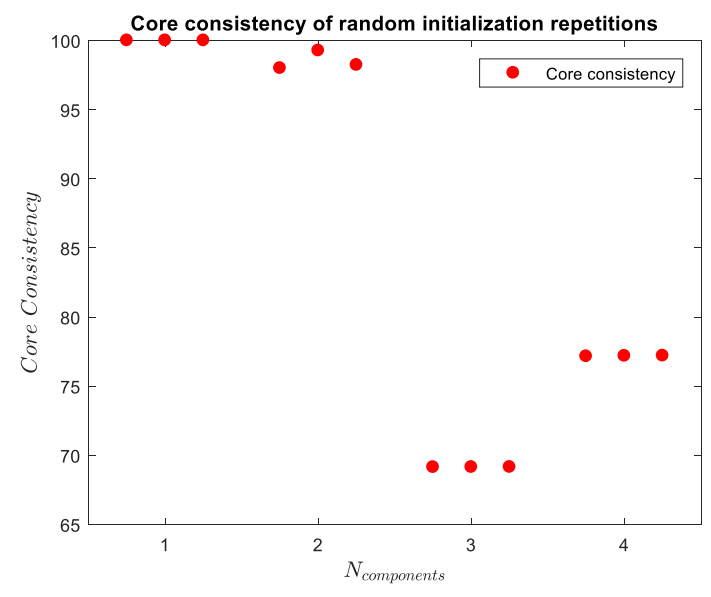

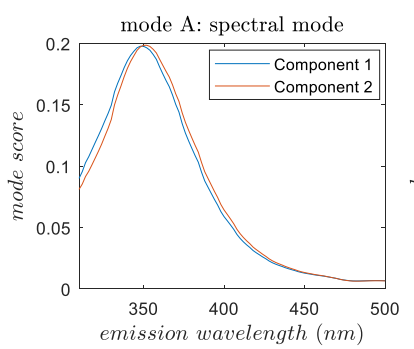

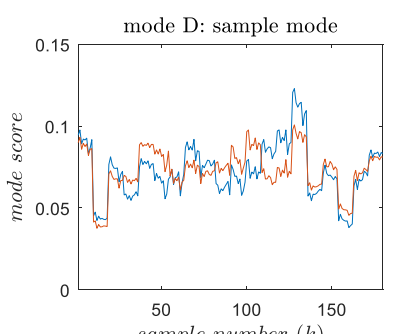

sample number $(k)$
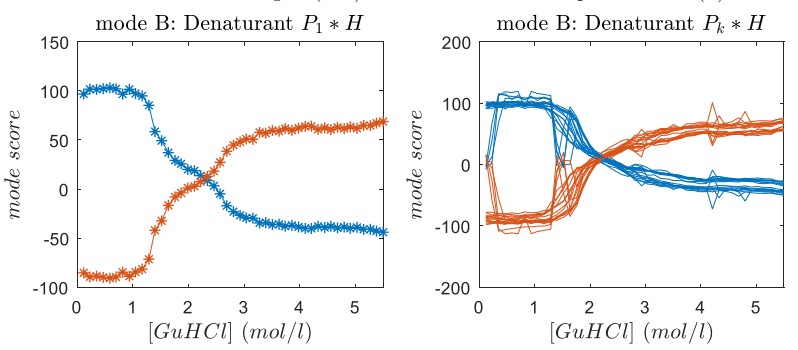

Figure S 2-4: Shows the results of PARAFAC2 modelling for the combined proteins from the $1^{\text {st }}$ experimental series. A) the core consistency as a function of number of components, B) all modes of the PARAFAC2 model. Only the first 27 transitions (PPI-01) are shown on the bottom right. $A$ total of $8.3 \%$ outliers was found in this model.

\section{S2.2.2 2nd Experimental screening}

A

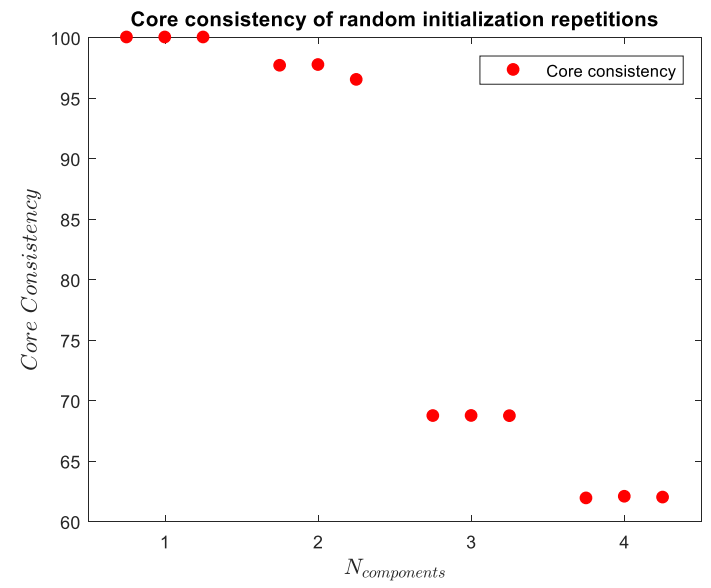

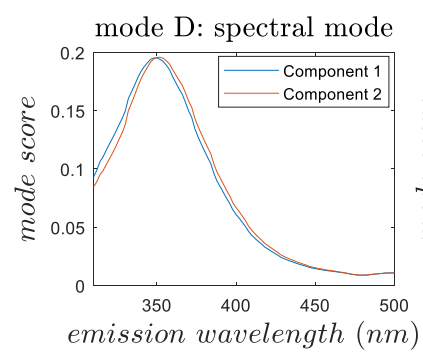

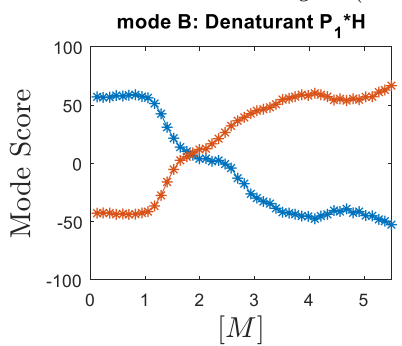

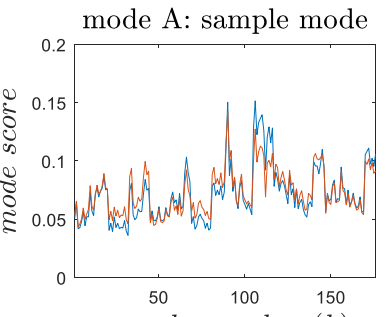

sample number $(k$ mode B: Denaturant $\mathbf{P}_{\mathbf{k}}{ }^{*} \mathbf{H}$

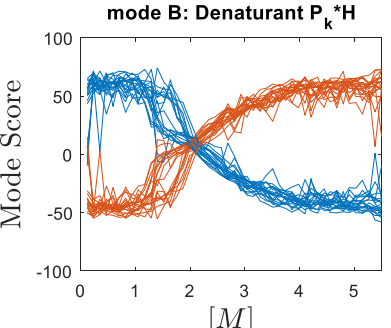

Figure S 2-5: Shows the results of PARAFAC2 modelling for the combined proteins from the $2^{\text {nd }}$ experimental series. A) the core consistency as a function of number of components, B) all modes of the PARAFAC2 model. Only the first 27 transitions (PPI-01) are shown on the bottom right. $A$ total of $8.6 \%$ outliers was found in this model. 
S3 Correction of modelled transition curves

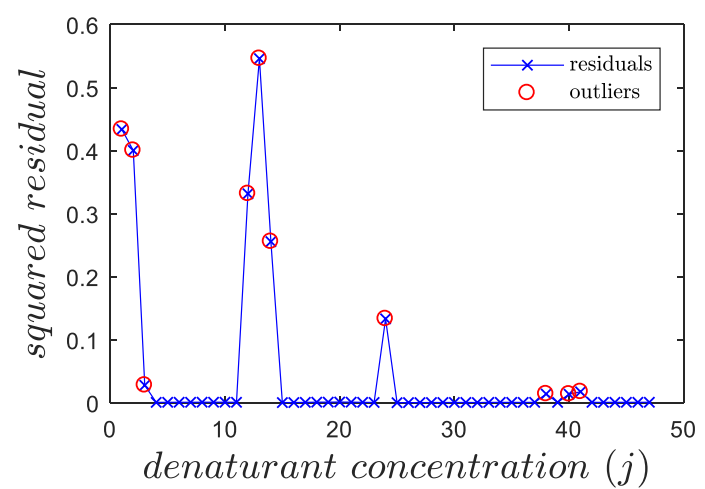

Figure S 3-1: Squared residuals plotted against the denaturant concentration for PPI-01, $\mathrm{pH} 7.5$ and $0 \mathrm{mM} \mathrm{NaCl}$. This figure is the one that is used to find the outliers indicated in Figure 10 in the manuscript. 